\title{
Agradecimento aos Revisores de Ciência \& Educação 2018, 2019, e jan.-set. 2020
}

\author{
Acknowledgement to Reviewers of Ciência \& Educação \\ in 2018, 2019, and Jan.-Sep. 2020
}

Ciência \& Educação, editada desde 1995, constituiu, ao longo dos seus 25 anos de ininterrupto funcionamento, um quadro de 665 avaliadores, todos pesquisadores com o título mínimo de doutor e com produção nas áreas de ensino de Ciências, Matemática e outras disciplinas correlatas. Essa equipe, bem como os corpos editoriais nacional, internacional e a equipe técnica, têm sido fundamentais para conduzir a revista ao posto de um dos principais periódicos na área, ocupando lugar de destaque nas avaliações de órgãos de pesquisa e de ensino no Brasil, e em diversos outros países onde se encontram nossos colaboradores, revisores, autores e leitores.

Ao nomear nas próximas páginas os pesquisadores que participaram da revisão de artigos dos últimos três anos, Ciência \& Educação agradece a valiosa colaboração de todos os revisores que dedicaram seu tempo e expertise ao rigoroso processo editorial deste periódico nas últimas décadas.

Informamos, ainda, que foram publicados um total de 120 artigos nos anos de 2018 e 2019 em conjunto, e, em 2020, com a adoção do formato de edição em fluxo contínuo, foram publicados mais de 65 manuscritos.

Ciência \& Educação, published since 1995, has constituted over the 25 years of uninterrupted existence a team of 665 evaluators, all researchers with a minimum PhD degree and with production in the areas of Science, Mathematics Education and other related fields. This board, together with the national, international editorial boards, and the technical team, have been essential in leading Ciência \& Educação to the post of one of the main journals in the field, occupying a prominent place in the evaluations by agencies dedicated to research and teaching both in Brazil and in a number of countries where our collaborators, authors and readers are based.

By naming the researchers who have participated in the review of articles over the last three years, Ciência \& Educação wishes to thank the valuable collaboration of all reviewers who have dedicated their time and expertise to the rigorous editorial process of this journal. We highlight that, in 2018 and 2019, a total of 120 articles were published by the journal. In 2020, with the adoption of the continuous flow edition format, over 65 manuscripts have been published. 


\section{8}

Abib, Maria L. V. S.

Almeida, Maria José

Almouloud, Saddo

Alves, João

Amador, Filomena

Andrade, Mariana A. B. S.

Angotti, José

Arruda, Sergio

Arteta Vargas, Judith

Assis, Alice

Auler, Décio

Auth, Milton

Ayres, Ana C. B. M.

Barchi, Rodrigo

Barros, Marcelo A.

Basso, Sabrina

Bastos Filho, Jenner

Bastos, Fernando

Bego, Amadeu

Bicudo, Maria A. V.

Bisch, Sérgio

Bizerra, Alessandra

Borges, Regina M. R.

Cachapuz, António

Calazans, Alex

Caldeira, Ana M. A.

Camargo, Éder P.

Camargo, Tatiana S.

Camino, Néstor

Campos, Luciana M. L.

Canavarro Benite, Anna

Carniatto, Irene

Carvalho, Carlos V. A.

Castiblanco, Olga L.

Cavassan, Osmar

Chapani, Daisi T.

Chrispino, Alvaro

Cirino, Marcelo M.

Coelho, Jonas G.

Compiani, Mauricio

Corrêa, André L.

Cortela, Beatriz S. C.

Couto, Cecília G.

Cunha, Marcia B.

D'Ambrosio, Ubiratan
Universidade de São Paulo, São Paulo, SP, Brasil

Universidade Estadual de Campinas, Campinas, SP, Brasil

Pontifícia Universidade Católica de São Paulo, São Paulo, SP, Brasil

Universidade Tecnológica Federal do Paraná, Curitiba, PR, Brasil

Universidade Aberta, Lisboa, Portugal

Universidade Estadual de Londrina, Londrina, PR, Brasil

Universidade Federal de Santa Catarina, Florianópolis, SC, Brasil

Universidade Estadual de Londrina, Londrina, PR, Brasil

Universidade del Norte, Barranquilla, Colombia

Universidade Estadual Paulista, Guaratinguetá, SP, Brasil

Universidade Federal de Santa Maria, Santa Maria, RS, Brasil

Universidade Federal de Uberlândia, Ituiutaba, MG, Brasil

Universidade do Estado do Rio de Janeiro, Rio de Janeiro, RJ, Brasil

Universidade de Sorocaba, Sorocaba, SP, Brasil

Universidade de São Paulo, São Carlos, SP, Brasil

Universidade Estadual Paulista, Bauru, SP, Brasil

Universidade Federal de Alagoas, Maceió, AL, Brasil

Universidade Estadual Paulista, Bauru, SP, Brasil

Universidade Estadual Paulista, Araraquara, SP, Brasil

Universidade Estadual Paulista, Rio Claro, SP, Brasil

Universidade Federal do Espírito Santo, Vitória, ES, Brasil

Universidade de São Paulo, São Paulo, SP, Brasil

Pontifícia Universidade Católica do Rio Grande do Sul, Porto Alegre, RS, Brasil

Universidade de Aveiro, Aveiro, Portugal

Universidade Tecnológica Federal do Paraná, Curitiba, PR, Brasil

Universidade Estadual Paulista, Bauru, SP, Brasil

Universidade Estadual Paulista, Ilha Solteira, SP, Brasil

Universidade Federal do Rio Grande do Sul, Porto Alegre, RS, Brasil

Consejo Nacional de Investigaciones Científicas y Técnicas, Chubut, Argentina

Universidade Estadual Paulista, Botucatu, SP, Brasil

Universidade Federal de Goiás, Goiânia, GO, Brasil

Universidade Estadual do Oeste do Paraná, Cascavel, PR, Brasil

Centro Universitário Estadual da Zona Oeste, Rio de Janeiro, RJ, Brasil

Universidad Distrital Francisco Jose de Caldas, Bogotá, Colombia

Universidade Estadual Paulista, Bauru, SP, Brasil

Universidade Estadual do Sudoeste da Bahia, Jequié, BA, Brasil

Centro Federal de Educação Tecnológica Celso S. da Fonseca, Rio de Janeiro, RJ, Brasil

Universidade Estadual de Londrina, Londrina, PR, Brasil

Universidade Estadual Paulista, Bauru, SP, Brasil

Universidade Estadual de Campinas, Campinas, SP, Brasil

Universidade Estadual de Santa Cruz, Ilhéus, BA, Brasil

Universidade Estadual Paulista, Bauru, SP, Brasil

Universidade de Lisboa, Lisboa, Portugal

Universidade Estadual do Oeste do Paraná, Toledo, PR, Brasil

Universidade Estadual de Campinas, Campinas, SP, Brasil 


\section{8}

Delizoicov Neto, Demétrio

Dias, Ana L. B.

Duarte, Ana C. S.

Echeverría, Agustina

El-Hani, Charbel

Farias, Carmen R. O.

Feltrin, Rebeca B.

Fernandes, Priscila C.

Ferreira, Ana C.

Ferreira, Juliana M. H.

Figueiredo, Adriana M.

Francisco Junior, Wilmo

Freitas, Zulind L.

Galiazzi, Maria C.

Galieta, Tatiana

Gonçalves, Fábio P.

Gonçalves, Pedro W.

Guimarães, Gilda L.

Guimarães, Márcio A.

Guimarães, Orliney

Gurgel, Célia

Hayashi, Maria

Hornink, Gabriel

Horvath, Jorge

Igliori, Sonia

Jacobi, Pedro

Jesus, Andréa C. S.

Justina, Lourdes A.

Kantor, Carlos

Kato, Danilo

Kiouranis, Neide

Klüber, Tiago

Lamas, Adriana

Lambach, Marcelo

Leitão, Carlos A. E.

Leite, Laurinda S. F.

Lima Junior, Paulo

Lindemann, Renata

Longhini, Marcos

Lopes, Celi E.

Lopes, Nataly C.

Lorencini Júnior, Álvaro

Loureiro, Carlos F. B.

Luz, Mauricio R. M. P.

Maia, Jorge S. S.
Universidade Federal de Santa Catarina, Florianópolis, SC, Brasil

Central Michigan University, Mount Pleasant, USA

Universidade Estadual do Sudoeste da Bahia, Jequié, BA, Brasil

Universidade Federal de Goiás, Goiânia, GO, Brasil

Universidade Federal da Bahia, Salvador, BA, Brasil

Universidade Federal Rural de Pernambuco, Recife, PE, Brasil

Universidade Estadual de Campinas, Campinas, SP, Brasil

Universidade Federal de Sao João del-Rei, São João del-Rei, MG, Brasil

Universidade Federal de Ouro Preto, Ouro Preto, MG, Brasil

Universidade Federal do Rio Grande do Norte, Natal, RN, Brasil

Universidade Federal de Ouro Preto, Ouro Preto, MG, Brasil

Universidade Federal de Alagoas, Arapiraca, AL, Brasil

Universidade Estadual Paulista, Ilha Solteira, SP, Brasil

Universidade Federal do Rio Grande, Rio Grance, RS, Brasil

Universidade do Estado do Rio de Janeiro, São Gonçalo, RJ, Brasil

Universidade Federal de Santa Catarina, Florianópolis, SC, Brasil

Universidade Estadual de Campinas, Campinas, SP, Brasil

Universidade Federal de Pernambuco, Jaboatão dos Guararapes, PE, Brasil

Universidade Federal de Sergipe, São Cristóvão, SE, Brasil

Universidade Federal do Paraná, Curitiba, PR, Brasil

Universidade Metodista, Piracicaba, SP, Brasil

Universidade Federal de Sao Carlos, São Carlos, SP, Brasil

Universidade Federal de Alfenas, Alfenas, MG, Brasil

Universidade de São Paulo, São Paulo, SP, Brasil

Pontifícia Universidade Católica de São Paulo, São Paulo, SP, Brasil

Universidade de São Paulo, São Paulo, SP, Brasil

Universidade Estadual Paulista, Bauru, SP, Brasil

Universidade Estadual do Oeste do Paraná, Cascavel, PR, Brasil

Centro Universitário Fundação Santo André, Santo André, SP, Brasil

Universidade Federal do Triângulo Mineiro, Uberaba, MG, Brasil

Universidade Estadual de Maringá, Maringá, MG, Brasil

Universidade Estadual do Oeste do Paraná, Cascavel, PR, Brasil

Universidade Federal do $A B C$, Santo André, SP, Brasil

Universidade Tecnológica Federal do Paraná, Curitiba, PR, Brasil

Universidade Estadual do Sudoeste da Bahia, Vitória da Conquista, BA, Brasil

Universidade do Minho, Braga, Portugal

Universidade de Brasília, Brasília, DF, Brasil

Universidade Federal do Pampa, Bagé, RS, Brasil

Universidade Federal de Uberlândia, Uberlândia, MG, Brasil

Universidade Cruzeiro do Sul, São Paulo, SP, Brasil

Universidade Federal de São Carlos, Araras, SP, Brasil

Universidade Estadual de Londrina, Londrina, PR, Brasil

Universidade Federal do Rio de Janeiro, Rio de Janeiro, RJ, Brasil

Fundação Oswaldo Cruz, Rio de Janeiro, RJ, Brasil

Universidade Estadual do Norte do Paraná, Jacarezinho, PR, Brasil 


\section{8}

Maia, Poliana F.

Maldaner, Otavio

Malheiro, João M. S.

Marques, Valéria

Massabni, Vânia

Massena, Elisa

Massi, Luciana

Megid Neto, Jorge

Meglhioratti, Fernanda

Mendes, Iran

Menezes, Paulo

Messeder Neto, Hélio

Mocellin, Ronei

Mohr, Adriana

Montanher, Valter

Monteiro, Maria A.

Mortimer, Eduardo

Motta, Micheline

Muenchen, Cristiane

Muñoz Albarracín, Liz

Nascimento Júnior, Antônio

Nascimento, Silvania

Nicolli, Aline

Obara, Ana

Oliveira, Eniz

Oliveira, Rosemary R.

Oliveira, Silmara S.

Oliveira, Vera L. B.

Orquiza-de-Carvalho, Lizete

Ortigão, Maria I. R.

Ostermann, Fernanda

Ovigli, Daniel

Paixão, Maria F. C. S.

Passos, Marinez

Pedrini, Alexandre

Pérez Miranda, Royman

Pierson, Alice

Pinhão, Francine

Pinheiro, Paulo $C$.

Pinto Neto, Pedro

Porro, Silvia

Prata, Rita

Prestes, Maria E. B.

Queirós, Wellington

Queiroz, Gloria
Universidade Federal de Viçosa, Florestal, MG, Brasil

Universidade Regional do Noroeste do Estado do Rio Grande do Sul, Ijuí, RS, Brasil Universidade Federal do Pará, Castanhal, PA, Brasil

Universidade Federal Rural do Rio de Janeiro, Seropédica, RJ, Brasil

Universidade de São Paulo, Piracicaba, SP, Brasil

Universidade Estadual de Santa Cruz, Ilhéus, BA, Brasil

Universidade Estadual Paulista, Araraquara, SP, Brasil

Universidade Estadual de Campinas, Campinas, SP, Brasil

Universidade Estadual do Oeste do Paraná, Cascavel, PR, Brasil

Universidade Federal do Pará, Belém, PA, Brasil

Universidade Federal de Juiz de Fora, Juiz de Fora, MG, Brasil

Universidade Federal da Bahia, Salvador, BA, Brasil

Universidade Tecnológica Federal do Paraná, Curitiba, PR, Brasil

Universidade Federal de Santa Catarina, Florianópolis, SC, Brasil

Instituto Federal de Educação, Ciência e Tecnologia de SP, Piracicaba, SP, Brasil

Universidade Federal da Grande Dourados, Dourados, MS, Brasil

Universidade Federal de Minas Gerais, Belo Horizonte, MG, Brasil

Universidade Federal de Pernambuco, Recife, PE, Brasil

Universidade Federal de Santa Maria, Santa Maria, RS, Brasil

Universidad Distrital Francisco Jose de Caldas, Bogotá, Colombia

Universidade Federal de Lavras, Lavras, MG, Brasil

Universidade Federal de Minas Gerais, Belo Horizonte, MG, Brasil

Universidade Federal do Acre, Rio Branco, AC, Brasil

Universidade Estadual de Maringá, Maringá, PR, Brasil

Centro Universitário Univates, Lajeado, RS, Brasil

Universidade Estadual Paulista, Jaboticabal, SP, Brasil

Universidade Estadual de Londrina, Londrina, PR, Brasil

Universidade Estadual de Londrina, Londrina, PR, Brasil

Universidade Estadual Paulista, Ilha Solteira, SP, Brasil

Universidade do Estado do Rio de Janeiro, Rio de Janeiro, RJ, Brasil

Universidade Federal do Rio Grande do Sul, Porto Alegre, RS, Brasil

Universidade Federal do Triângulo Mineiro, Uberaba, MG, Brasil

Instituto Politécnico de Castelo Branco, Castelo Branco, Portugal

Universidade Estadual de Londrina, Londrina, PR, Brasil

Universidade do Estado do Rio de Janeiro, Rio de Janeiro, RJ, Brasil

Universidad Pedagógica Nacional, Bogotá, Colombia

Universidade Federal de São Carlos, São Carlos, SP, Brasil

Universidade do Estado do Rio de Janeiro, São Gonçalo, RJ, Brasil

Universidade Federal de São João del-Rei, São João del-Rei, MG, Brasil

Universidade Estadual de Campinas, Campinas, SP, Brasil

Universidad Nacional de Quilmes, Bernal, Argentina

Universidade Federal do Rio de Janeiro, Rio de Janeiro, RJ, Brasil

Universidade de São Paulo, São Paulo, SP, Brasil

Universidade Federal de Mato Grosso do Sul, Campo Grande, MS, Brasil

Universidade do Estado do Rio de Janeiro, Rio de Janeiro, RJ, Brasil 


\section{8}

Quintanilla-Gatica, Mario

Razera, Júlio

Recena, Maria

Rezende, Flávia

Rezende Filho, Luiz

Rosa, Katemari

Rosa, Paulo R. S.

Rothberg, Danilo

Salazar Lopez, Tatiana

Salvi, Rosana

Sampaio, Shaula

Santos, André L. T. L.

Santos, Bruno F.

Santos, Paulo G. F.

Silva, Adjane C. T.

Silva, Alcina M. T. B.

Silva, Amarildo M.

Silva, Antonio F. G.

Silva, Camila S.

Silva, Dirceu

Silva, Henrique C.

Silva, Jackson G.

Silva, Maria G. V.

Sisla, Heloisa

Sobreira, Paulo

Sousa, Guaracira G.

Sousa, Isabela C. F.

Souza, Aguinaldo R.

Souza, Daniele C.

Souza, Marcos L.

Souza, Thais S. N.

Sovierzoski, Hilda H.

Spazziani, Maria

Strieder, Roseline

Struchiner, Miriam

Suart Júnior, José

Talamoni, Ana

Talamoni, Jandira

Teixeira, Lucas A.

Teixeira, Odete $P$.

Teixeira, Paulo M. M.

Toti, Frederico

Utsumi, Miriam

Valdemarin, Vera

Valente, Jose Armando
Pontificia Universidad Católica de Chile, Santiago, Chile

Universidade Estadual do Sudoeste da Bahia, Jequié, BA, Brasil

Universidade Federal de Mato Grosso do Sul, Campo Grande, MS, Brasil

Universidade Federal do Rio Grande do Sul, Porto Alegre, RS, Brasil

Universidade Federal do Rio de Janeiro, Rio de Janeiro, RJ, Brasil

Universidade Federal da Bahia, Salvador, BA, Brasil

Universidade Federal de Mato Grosso do Sul, Campo Grande, MS, Brasil

Universidade Estadual Paulista, Bauru, SP, Brasil

Universidad del Valle, Cali, Colombia

Universidade Estadual de Londrina, Londrina, PR, Brasil

Universidade Federal Fluminense, Niterói, RJ, Brasil

Colégio Pedro II, Rio de Janeiro, RJ, Brasil

Universidade Estadual do Sudoeste da Bahia, Jequié, BA, Brasil

Universidade de Brasília, Planaltina, DF, Brasil

Universidade Federal de Sergipe, São Cristóvão, SE, Brasil

Centro Federal de Educação Tecnológica C. S. da Fonseca, Rio de Janeiro, RJ, Brasil

Universidade Federal de Juiz de Fora, Juiz de Fora, MG, Brasil

Universidade Federal de São Carlos, São Carlos, SP, Brasil

Universidade Federal do Paraná, Curitiba, PR, Brasil

Universidade Estadual de Campinas, Campinas, SP, Brasil

Universidade Federal de Santa Catarina, Florianópolis, SC, Brasil

Universidade Estadual Paulista, São José do Rio Preto, SP, Brasil

Universidade Federal do Ceará, Fortaleza, CE, Brasil

Universidade Federal de Sao Carlos, São Carlos, SP, Brasil

Universidade Federal de Goiás, Goiânia, GO, Brasil

Universidade Federal do Rio de Janeiro, Rio de Janeiro, RJ, Brasil

Fundação Oswaldo Cruz, Rio de Janeiro, RJ, Brasil

Universidade Estadual Paulista, Bauru, SP, Brasil

Universidade Federal do Triângulo Mineiro, Uberaba, MG, Brasil

Universidade Estadual do Sudoeste da Bahia, Jequié, BA, Brasil

Universidade Federal do Estado do Rio de Janeiro, Rio de Janeiro, RJ, Brasil

Universidade Federal de Alagoas, Maceió, AL, Brasil

Universidade Estadual Paulista, Botucatu, SP, Brasil

Universidade de Brasília, Brasília, DF, Brasil

Universidade Federal do Rio de Janeiro, Rio de Janeiro, RJ, Brasil

Universidade Tecnológica Federal do Paraná, Apucarana, PR, Brasil

Universidade Estadual Paulista, São Vicente, SP, Brasil

Universidade Estadual Paulista, Bauru, SP, Brasil

Universidade Estadual Paulista, Araraquara, SP, Brasil

Universidade Estadual Paulista, Guaratinguetá, SP, Brasil

Universidade Estadual do Sudoeste da Bahia, Jequi, BA, Brasil

Universidade Federal de Alfenas, Alfenas, MG, Brasil

Universidade de São Paulo, São Carlos, SP, Brasil

Universidade Estadual Paulista, Araraquara, SP, Brasil

Universidade Estadual de Campinas, Campinas, SP, Brasil 
2018

\author{
Vaz da Silva, Arnaldo \\ Veraszto, Estéfano \\ Vertuan, Rodolfo \\ Vianna, Carlos R. \\ Vianna, Deise M. \\ Viveiro, Alessandra \\ Vizcaino Arevalo, Diego \\ Zanata, Eliana \\ Zanon, Lenir \\ Zompero, Andreia \\ Zuliani, Silvia
}

Universidade Federal de Minas Gerais, Belo Horizonte. MG, Brasil

Universidade Federal de São Carlos, São Carlos, SP, Brasil

Universidade Tecnologica Federal do Paraná, Toledo, PR, Brasil

Universidade Federal do Paraná, Curitiba, PR, Brasil

Universidade Federal do Rio de Janeiro, Rio de Janeiro, RJ, Brasil

Universidade Estadual de Campinas, Campinas, SP, Brasil

Universidad Distrital Francisco Jose de Caldas, Bogotá, Colombia

Universidade Estadual Paulista, Bauru, SP, Brasil

Universidade Regional do Noroeste do Estado do Rio Grande do Sul, Ijui, RS, Brasil

Universidade Norte do Paraná, Curitiba, PR, Brasil

Universidade Estadual Paulista, Bauru, SP, Brasil

\section{9}

Albrecht, Evonir

Almeida, Lourdes M. L.

Alves Filho, José P.

Amador, Filomena

Amorim, Antonio C. R.

Andrade, Mariana A. B. S.

Andrade, Silvanio

Araujo Neto, Waldmir

Araújo, Mauro S. T.

Arruda, Sergio

Assis, Alice

Auler, Décio

Barchi, Rodrigo

Barros, Daniela M. V.

Barzano, Marco

Bastos Filho, Jenner

Bastos, Fernando

Batinga, Verônica

Batista, Sylvia H. S. S.

Bayer, Arno

Bego, Amadeu

Bernardes, Maria E. M.

Bisch, Sérgio

Bizerril, Marcelo

Bomfim, Alexandre

Bonzanini, Taitiany K.

Bortoletto, Adriana

Bretones, Paulo S.

Buriasco, Regina

Caldeira, Ana M. A.

Camargo Filho, Paulo S.
Universidade Federal do $A B C$, Santo André, SP, Brasil

Universidade Estadual de Londrina, Londrina, PR, Brasil

Universidade Federal de Santa Catarina, Florianópolis, SC, Brasil

Universidade Aberta, Lisboa, Portugal

Universidade Estadual de Campinas, Campinas, SP, Brasil

Universidade Estadual de Londrina, Londrina, PR, Brasil

Universidade Estadual da Paraiba, Campina Grande, PB, Brasil

Universidade Federal do Rio de Janeiro, Rio de Janeiro, RJ, Brasil

Universidade Cruzeiro do Sul, São Paulo, SP, Brasil

Universidade Estadual de Londrina, Londrina, PR, Brasil

niversidade Estadual Paulista, Guaratinguetá, SP, Brasil

Universidade Federal de Santa Maria, Santa Maria, RS, Brasil

Universidade de Sorocaba, Sorocaba, SP, Brasil

Universidade Aberta, Lisboa, Portugal

Universidade Estadual de Feira de Santana, Feira de Santana, BA, Brasil

Universidade Federal de Alagoas, Maceió, AL, Brasil

Universidade Estadual Paulista, Bauru, SP, Brasil

Universidade Federal de Pernambuco, Recife, PE, Brasil

Universidade Federal de São Paulo, São Paulo, SP, Brasil

Universidade Luterana do Brasil, Canoas, RS, Brasil

Universidade Estadual Paulista, Araraquara, SP, Brasil

Universidade de São Paulo, São Paulo, SP, Brasil

Universidade Federal do Espírito Santo, Vitória, ES, Brasil

Universidade de Brasília, Brasília, DF, Brasil

Instituto Federal de Educação, Ciência e Tecnologia do Rio de Janeiro, Nilópolis, RJ, Brasil

Universidade de São Paulo, Piracicaba, SP, Brasil

Universidade Estadual Paulista, Ilha Solteira, SP, Brasil

Universidade Federal de São Carlos, São Carlos, SP, Brasil

Universidade Estadual de Londrina, Londrina, PR, Brasil

Universidade Estadual Paulista, Bauru, SP, Brasil

Universidade Tecnológica Federal do Paraná, Londrina, PR, Brasil 


\section{9}

Camargo, Éder P.

Camargo, Tatiana S.

Camillo, Juliano

Campos, Luciana M. L.

Canavarro Benite, Anna

Candela Martín, Antonia

Carniatto, Irene

Carvalho, Carlos V. A.

Carvalho, Luis M.

Cavalari, Rosa

Cavalcanti, Eduardo

Cavassan, Osmar

Chapani, Daisi T.

Chassot, Attico

Cirino, Marcelo $\mathrm{M}$.

Coelho, Jonas G.

Compiani, Mauricio

Corrêa, André L.

Cortela, Beatriz S. C.

Costa, Nielce M. L.

Cribb, Sandra

Cunha, Ana M. O.

Cunha, Marcia B.

D'Ambrosio, Ubiratan

Del Pino, José

Delizoicov, Nadir C.

Dias, Ana L. B.

Dominguez, Celi

Duarte, Ana C. S.

Dynnikov, Circe

Eichler, Marcelo

Feltrin, Rebeca B.

Ferraro Junior, Luiz A.

Ferreira, Ana C.

Ferreira, Gabriela K.

Ferreira, Juliana M. H.

Figueiredo, Adriana M.

Figueirôa, Silvia

Firme, Ruth

Fonseca, Alexandre B. C.

Freire Junior, Olival

Freitas, Denise

Galembeck, Eduardo

Garcia, Nilson

Gehlen, Simoni
Universidade Estadual Paulista, Ilha Solteira, SP, Brasil

Universidade Federal do Rio Grande do Sul, Porto Alegre, RS, Brasil

Universidade Federal de Santa Catarina, Florianópolis, SC, Brasil

Universidade Estadual Paulista, Botucatu, SP, Brasil

Universidade Federal de Goiás, Goiânia, GO, Brasil

Instituto Politécnico Nacional, Ciudad de México, México

Universidade Estadual do Oeste do Paraná, Cascavel, PR, Brasil

Centro Universitário Estadual da Zona Oeste, Rio de Janeiro, RJ, Brasil

Universidade Estadual Paulista, Rio Claro, SP, Brasil

Universidade Estadual Paulista, Rio Claro, SP, Brasil

Universidade de Brasília, Brasília, DF, Brasil

Universidade Estadual Paulista, Bauru, SP, Brasil

Universidade Estadual do Sudoeste da Bahia, Jequié, BA, Brasil

Centro Universitário Metodista, Porto Alegre, RS, Brasil

Universidade Estadual de Londrina, Londrina, PR, Brasil

Universidade Estadual Paulista, Bauru, SP, Brasil

Universidade Estadual de Campinas, Campinas, SP, Brasil

Universidade Estadual de Santa Cruz, Ilhéus, BA, Brasil

Universidade Estadual Paulista, Bauru, SP, Brasil

Universidade Anhanguera de São Paulo, São Paulo, SP, Brasil

Centro Universitário Augusto Motta, Rio de Janeiro, RJ, Brasil

Universidade Federal de Uberlândia, Uberlândia, MG, Brasil

Universidade Estadual do Oeste do Paraná, Toledo, PR, Brasil

Universidade Estadual de Campinas, Campinas, SP, Brasil

Universidade Federal do Rio Grande do Sul, Porto Alegre, RS, Brasil

Universidade Comunitária da Região de Chapecó, Chapecó, RS, Brasil

Central Michigan University, Mount Pleasant, USA

Universidade de São Paulo, São Paulo, SP, Brasil

Universidade Estadual do Sudoeste da Bahia, Jequié, BA, Brasil

Universidade Federal do Espírito Santo, Vitória, ES, Brasil

Universidade Federal do Rio Grande do Sul, Porto Alegre, RS, Brasil

Universidade Estadual de Campinas, Campinas, SP, Brasil

Universidade Estadual de Feira de Santana, Feira de Santana, BA, Brasil

Universidade Federal de Ouro Preto, Ouro Preto, MG, Brasil

Universidade Federal do Paraná, Curitiba, PR, Brasil

Universidade Federal do Rio Grande do Norte, Natal, RN, Brasil

Universidade Federal de Ouro Preto, Ouro Preto, MG, Brasil

Universidade Estadual de Campinas, Campinas, SP, Brasil

Universidade Federal Rural de Pernambuco, Recife, PE, Brasil

Universidade Federal do Rio de Janeiro, Rio de Janeiro, RJ, Brasil

Universidade Federal da Bahia, Salvador, BA, Brasil

Universidade Federal de São Carlos, São Carlos, SP, Brasil

Universidade Estadual de Campinas, Campinas, SP, Brasil

Universidade Tecnológica Federal do Paraná, Curitiba, PR, Brasil

Universidade Estadual de Santa Cruz, Ilhéus, BA, Brasil 


\section{9}

Ghilardi-Lopes, Natalia

Giannella, Tais

Gonçalves, Fábio P.

Gonçalves, Pedro W.

Gonçalves, Terezinha V. O.

Guido, Lucia

Gusmão, Tania

lachel, Gustavo

Jacobi, Pedro

Jesus, Andréa C. S,

Kato, Danilo

Labarce, Eliane

Laburú, Carlos

Lambach, Marcelo

Layrargues, Philippe

Leitão, Carlos A. E.

Leite, Cristina

Leite, Laurinda S. F.

Lima Junior, Paulo

Lima, Sorandra

Lima, Valderez M. R.

Longhini, lara

Lopes, Nataly C.

Lorencini Júnior, Álvaro

Luz, Mauricio R. M. P.

Maia, Jorge S. S.

Maio, Eliane R.

Maldaner, Otavio

Marandino, Martha

Marcondes, Maria E. R.

Maricato, Fúlvia

Marques, Carlos A.

Marques, Joana B. V.

Marques, Valéria

Martins-Salandim, Maria E.

Massi, Luciana

Matos, Mauricio S.

Megid Neto, Jorge

Meglhioratti, Fernanda

Meneses Villagrá, Jesus

Messeder Neto, Hélio

Michinel, José

Mocellin, Ronei

Monroy-Nasr, Zuraya

Monteiro, Maria A.
Universidade Federal do $A B C$, Santo André, SP, Brasil

Universidade Federal do Rio de Janeiro, Rio de Janeiro, RJ, Brasil

Universidade Federal de Santa Catarina, Florianópolis, SC, Brasil

Universidade Estadual de Campinas, Campinas, SP, Brasil

Universidade Federal do Pará, Belém, PA, Brasil

Universidade Federal de Uberlândia, Uberlândia, MG, Brasil

Universidade Estadual do Sudoeste da Bahia, Vitória da Conquista, BA, Brasil

Universidade Estadual de Londrina, Londrina, PR, Brasil

Universidade de São Paulo, São Paulo, SP, Brasil

Universidade Estadual Paulista, Bauru, SP, Brasil

Universidade Federal do Triângulo Mineiro, Uberaba, MG, Brasil

Universidade Estadual do Mato Grosso do Sul, Coxim, MS, Brasil

Universidade Estadual de Londrina, Londrina, PR, Brasil

Universidade Tecnológica Federal do Paraná, Curitiba, PR, Brasil

Universidade de Brasilia, Brasília, DF, Brasil

Universidade Estadual do Sudoeste da Bahia, Vitória da Conquista, BA, Brasil

Universidade de São Paulo, São Paulo, SP, Brasil

Universidade do Minho, Braga, Portugal

Universidade de Brasília, Brasília, DF, Brasil

Universidade Federal de Uberlândia, Uberlândia, MG, Brasil

Pontificia Universidade Catolica do Rio Grande do Sul, Porto Alegre, RS, Brasil

Universidade Federal de Uberlândia, Uberlândia, MG, Brasil

Universidade Federal de São Carlos, Araras, SP, Brasil

Universidade Estadual de Londrina, Londrina, PR, Brasil

Fundação Oswaldo Cruz, Rio de Janeiro, RJ, Brasil

Universidade Estadual do Norte do Paraná, Jacarezinho, PR, Brasil

Universidade Estadual de Maringá, Maringá, PR, Brasil

Universidade Regional do Noroeste do Estado do Rio Grande do Sul, ljuí, RS, Brasil

Universidade de São Paulo, São Paulo, SP, Brasil

Universidade de São Paulo, São Paulo, SP, Brasil

Universidade Estadual de Maringá, Maringá, PR, Brasil

Universidade Federal de Santa Catarina, Florianópolis, SC, Brasil

Universidade Federal de São Carlos, São Carlos, SP, Brasil

Universidade Federal Rural do Rio de Janeiro, Seropédica, RJ, Brasil

Universidade Estadual Paulista, Baurullha Solteira, SP, Brasil

Universidade Estadual Paulista, Araraquara, SP, Brasil

Universidade de São Paulo, São Paulo, SP, Brasil

Universidade Estadual de Campinas, Campinas, SP, Brasil

Universidade Estadual do Oeste do Paraná, Cascavel, PR, Brasil

Universidad de Burgos, Burgos, España

Universidade Federal da Bahia, Salvador, BA, Brasil

Universidade Estadual de Feira de Santana, Feira de Santana, BA, Brasil

Universidade Tecnologica Federal do Parana, Curitiba, PR, Brasil

Universidad Nacional Autonoma de Mexico, Ciudad de Mexico, Mexico

Universidade Federal da Grande Dourados, Dourados, MS, Brasil 


\section{9}

Moreira, Plínio C.

Moretti, Méricles

Muñoz Albarracín, Liz

Nascimento Júnior, Antônio

Nascimento, Silvania

Nicolli, Aline

Oliveira, Andréia M. P.

Oliveira, Anna

Oliveira, Eniz

Oliveira, Silmara S.

Oliveira, Vera L. B.

Orquiza-de-Carvalho, Lizete

Ovigli, Daniel

Passos, Marinez

Pedrini, Alexandre

Pereira, Alexsandro

Pierson, Alice

Pietrocola, Maurício

Pinto Neto, Pedro

Porro, Silvia

Pralon, Lúcia

Queirós, Wellington

Queiroz, Gloria

Rabelo, Mauro

Raboni, Paulo

Razera, Júlio

Reigota, Marcos

Reis, Pedro G. R.

Rezende, Flavia

Rezende Junior, Mikael

Ritter, Jaqueline

Rodrigues, André M.

Rodrigues, Angélica C.

Rolkouski, Emerson

Rosa, Maria I. F. P. S.

Saito, Fumikazu

Salazar Lopez, Tatiana

Santos, Bruno F.

Santos, Paulo G. F.

Sasseron, Lucia

Sato, Michele

Silva, Alcina M. T. B.

Silva, Antonio F. G.

Silva, Cibelle

Silva, Dirceu

Silva, Jackson G.
Universidade Federal de Ouro Preto, Ouro Preto, MG, Brasil Universidade Federal de Santa Catarina, Florianópolis, SC, Brasil Universidad Distrital Francisco Jose de Caldas, Bogotá, Colombia Universidade Federal de Lavras, Lavras, MG, Brasil Universidade Federal de Minas Gerais, Belo Horizonte, MG, Brasil Universidade Federal do Acre, Rio Branco, AC, Brasil Universidade Federal da Bahia, Salvador, BA, Brasil Universidade Estadual Paulista, Marília, SP, Brasil Centro Universitário Univates, Lajeado, RS, Brasil Universidade Estadual de Londrina, Londrina, PR, Brasil Universidade Estadual de Londrina, Londrina, PR, Brasil Universidade Estadual Paulista, Ilha Solteira, SP, Brasil Universidade Federal do Triângulo Mineiro, Uberaba, MG, Brasil Universidade Estadual de Londrina, Londrina, PR, Brasil Universidade do Estado do Rio de Janeiro, Rio de Janeiro, RJ, Brasil Universidade Federal do Rio Grande do Sul, Porto Alegre, RS, Brasil Universidade Federal de São Carlos, São Carlos, SP, Brasil Universidade de São Paulo, São Paulo, SP, Brasil Universidade Estadual de Campinas, Campinas, SP, Brasil Universidad Nacional de Quilmes, Bernal, Argentina Universidade Federal do Estado do Rio de Janeiro, Rio de Janeiro, RJ, Brasil Universidade Federal de Mato Grosso do Sul, Campo Grande, MS, Brasil Universidade do Estado do Rio de Janeiro, Rio de Janeiro, RJ, Brasil Universidade de Brasilia, Brasília, DF, Brasil Universidade Estadual Paulista, Presidente Prudente, SP, Brasil Universidade Estadual do Sudoeste da Bahia, Jequié, BA, Brasil Universidade de Sorocaba, Sorocaba, SP, Brasil Universidade de Lisboa, Lisboa, Portugal Universidade Federal do Rio Grande do Sul, Porto Alegre, RS, Brasil Universidade Federal de Itajubá, Itajubá, MG, Brasil Universidade Federal do Rio Grande, Rio Grande, RS, Brasil Universidade de São Paulo, São Paulo, SP, Brasil Universidade Federal de Juiz de Fora, Juiz de Fora, MG, Brasil Universidade Federal do Paraná, Curitiba, PR, Brasil Universidade Estadual de Campinas, Campinas, SP, Brasil Pontifícia Universidade Católica de São Paulo, São Paulo, SP, Brasil CINVESTAV Monterrey, Ciudad de Mexico, Mexico Universidade Estadual do Sudoeste da Bahia, Jequié, BA, Brasil Universidade de Brasília, Planaltina, DF, Brasil Universidade de São Paulo, São Paulo, SP, Brasil Universidade Federal de Mato Grosso, Cuiabá, MT, Brasil Centro Federal de Educação Tecnológica C. Suckow da Fonseca, Rio de Janeiro, RJ, Brasil Universidade Federal de São Carlos, São Carlos, SP, Brasil Universidade de São Paulo, São Carlos, SP, Brasil Universidade Estadual de Campinas, Campinas, SP, Brasil Universidade Estadual Paulista, São José do Rio Preto, SP, Brasil 


\section{9}

Silva, João A.

Silva, Osmar H. M.

Silva, Sani C. R.

Siqueira, Maxwell

Siqueira, Vera

Sisla, Heloisa

Soares, Márlon

Sobreira, Paulo

Sousa, Isabela C. F.

Souza, Aguinaldo R.

Souza, Daniele C.

Souza, Francislê

Souza, Thais S. N.

Spazziani, Maria

Strieder, Dulce

Struchiner, Miriam

Suart Júnior, José

Talamoni, Ana C.

Teixeira, Lucas A.

Teixeira, Odete P.

Teixeira, Paulo M. M.

Testoni, Leonardo

Valadares, Jorge

Valente, Jose Armando

Vázquez Alonso, Angel

Venturi, Tiago

Veraszto, Estéfano

Vertuan, Rodolfo

Vianna, Carlos R.

Vianna, Deise M.

Vilela, Denise

Zanata, Eliana

Zompero, Andreia

Zuffi, Edna

Zuliani, Silvia
Universidade Federal do Rio Grande, Rio Grande, RS, Brasil

Universidade Estadual de Londrina, Londrina, PR, Brasil

Universidade Tecnológica Federal do Paraná, Ponta Grossa, PR, Brasil

Universidade Estadual de Santa Cruz, Ilhéus, BA, Brasil

Universidade Federal do Rio de Janeiro, Rio de Janeiro, RJ, Brasil

Universidade Federal de São Carlos, São Carlos, SP, Brasil

Universidade Federal de Goiás, Goiânia, GO, Brasil

Universidade Federal de Goiás, Goiânia, GO, Brasil

Fundação Oswaldo Cruz, Rio de Janeiro, RJ, Brasil

Universidade Estadual Paulista, Bauru, SP, Brasil

Universidade Federal do Triângulo Mineiro, Uberaba, MG, Brasil

Universidade de Aveiro, Aveiro, Portugal

Universidade Federal do Estado do Rio de Janeiro, Rio de Janeiro, RJ, Brasil Universidade Estadual Paulista, Botucatu, SP, Brasil

Universidade Estadual do Oeste do Paraná, Cascavel, PR, Brasil

Universidade Federal do Rio de Janeiro, Rio de Janeiro, RJ, Brasil

Universidade Tecnológica Federal do Paraná, Apucarana, PR, Brasil

Universidade Estadual Paulista, São Vicente, SP, Brasil

Universidade Estadual Paulista, Araraquara, SP, Brasil

Universidade Estadual Paulista, Guaratinguetá, SP, Brasil

Universidade Estadual do Sudoeste da Bahia, Jequi, BA, Brasil

Universidade Federal de São Paulo, Diadema, SP, Brasil

Universidade Aberta, Lisboa, Portugal

Universidade Estadual de Campinas, Campinas, SP, Brasil

Universitat de les Illes Balears, Palma, España

Universidade do Estado de Santa Catarina, Ibirama, SC, Brasil

Universidade Federal de São Carlos, São Carlos, SP, Brasil

Universidade Tecnologica Federal do Paraná, Toledo, PR, Brasil

Universidade Federal do Paraná, Curitiba, PR, Brasil

Universidade Federal do Rio de Janeiro, Rio de Janeiro, RJ, Brasil

Universidade Federal de São Carlos, São Carlos, SP, Brasil

Universidade Estadual Paulista, Bauru, SP, Brasil

Universidade Norte do Paraná, Londrina, PR, Brasil

Universidade de São Paulo, São Carlos, SP, Brasil

Universidade Estadual Paulista, Bauru, SP, Brasil

\section{Janeiro a Setembro de 2020}

Abranches, Sérgio

Adúriz-Bravo, Agustín

Almeida, Maria José

Alves, João

Amador, Filomena

Amorim, Antonio C. R.

Andrade, Mariana A. B. S.
Universidade Federal de Pernambuco, Recife, PE, Brasil

Universidad de Buenos Aires, Buenos Aires, Argentina

Universidade Estadual de Campinas, Campinas, SP, Brasil

Universidade Tecnológica Federal do Paraná, Curitiba, PR, Brasil

Universidade Aberta, Lisboa, Portugal

Universidade Estadual de Campinas, Campinas, SP, Brasil

Universidade Estadual de Londrina, Londrina, PR, Brasil 


\section{Janeiro a Setembro de 2020}

Anjos, Maylta

Arroio, Agnaldo

Arruda, Sergio

Arteta Vargas, Judith

Ayres, Ana C. B. M.

Bagdonas, Alexandre

Baraldi, Ivete

Barchi, Rodrigo

Barros, Daniela M. V.

Barzano, Marco

Bastos Filho, Jenner

Batista, Sylvia H. S. S.

Bayer, Arno

Bicudo, Maria A. V.

Biembengut, Maria

Bizerra, Alessandra

Bonzanini, Taitiany K.

Bozelli, Fernanda

Braga, Marco

Buriasco, Regina

Cabral, Tânia

Caldeira, Ana M. A.

Camargo, Éder P.

Camillo, Juliano

Candela Martín, Antonia

Cardia, Edson

Cardia, Elizabeth

Carniatto, Irene

Carnio, Michel

Carvalho, Carlos V. A.

Carvalho, Luis M.

Castiblanco, Olga L.

Cavassan, Osmar

Chapani, Daisi T.

Chassot, Attico

Chrispino, Alvaro

Cirino, Marcelo M.

Coelho, Jonas G.

Cortela, Beatriz S. C.

Cribb, Sandra

Cunha, Marcia B.

D'Ambrosio, Ubiratan

Del Pino, José

Delizoicov, Nadir C.

Dias, Ana L. B.
Instituto Federal de Educação Ciência e Tecnologia do Rio de Janeiro, Nilópolis, Rj, Brasil Universidade de São Paulo, São Paulo, SP, Brasil

Universidade Estadual de Londrina, Londrina, PR, Brasil

Universidade del Norte, Barranquilla, Colombia

Universidade do Estado do Rio de Janeiro, Rio de Janeiro, RJ, Brasil

Universidade Federal de Lavras, Lavras, MG, Brasil

Universidade Estadual Paulista, Bauru, SP, Brasil

Universidade de Sorocaba, Sorocaba, SP, Brasil

Universidade Aberta, Lisboa, Portugal

Universidade Estadual de Feira de Santana, Feira de Santana, BA, Brasil

Universidade Federal de Alagoas, Maceió, AL, Brasil

Universidade Federal de São Paulo, São Paulo, SP, Brasil

Universidade Luterana do Brasil, Canoas, RS, Brasil

Universidade Estadual Paulista, Rio Claro, SP, Brasil

Pontifícia Universidade Católica do Rio Grande do Sul, Porto Alegre, Rs, Brasil

Universidade de São Paulo, São Paulo, SP, Brasil

Universidade de São Paulo, Piracicaba, SP, Brasil

Universidade Estadual Paulista, Ilha Solteira, SP, Brasil

Centro Federal de Educação Tecnológica Celso S. da Fonseca, Rio de Janeiro, RJ, Brasil Universidade Estadual de Londrina, Londrina, PR, Brasil

Pontifícia Universidade Católica do Rio Grande do Sul, Porto Alegre, Rs, Brasil

Universidade Estadual Paulista, Bauru, SP, Brasil

Universidade Estadual Paulista, Ilha Solteira, SP, Brasil

Universidade Federal de Santa Catarina, Florianópolis, SC, Brasil

Instituto Politécnico Nacional, Ciudad de México, México

Instituto de Ensino Superior de Bauru, Bauru, SP, Brasil

Universidade do Sagrado Coração, Bauru, SP, Brasil

Universidade Estadual do Oeste do Paraná, Cascavel, PR, Brasil

Universidade Federal de São Carlos, São Carlos, SP, Brasil

Centro Universitário Estadual da Zona Oeste, Rio de Janeiro, RJ, Brasil

Universidade Estadual Paulista, Rio Claro, SP, Brasil

Universidad Distrital Francisco Jose de Caldas, Bogotá, Colombia

Universidade Estadual Paulista, Bauru, SP, Brasil

Universidade Estadual do Sudoeste da Bahia, Jequié, BA, Brasil

Centro Universitário Metodista, Porto Alegre, RS, Brasil

Centro Federal de Educação Tecnológica C. Suckow da Fonseca, Rio de Janeiro, RJ, Brasil

Universidade Estadual de Londrina, Londrina, PR, Brasil

Universidade Estadual Paulista, Bauru, SP, Brasil

Universidade Estadual Paulista, Bauru, SP, Brasil

Centro Universitário Augusto Motta, Rio de Janeiro, RJ, Brasil

Universidade Estadual do Oeste do Paraná, Toledo, PR, Brasil

Universidade Estadual de Campinas, Campinas, SP, Brasil

Universidade Federal do Rio Grande do Sul, Porto Alegre, RS, Brasil

Universidade Comunitária da Região de Chapecó, Chapecó, RS, Brasil

Central Michigan University, Mount Pleasant, USA 


\section{Janeiro a Setembro de 2020}

Dias, André L. M.

Duarte, Ana C. S.

Eichler, Marcelo

El-Hani, Charbel

Feltrin, Rebeca B.

Fernandez, Fernanda

Ferraro Junior, Luiz A.

Ferreira, Ana C.

Ferreira, Juliana M. H.

Figueirôa, Silvia

Forato, Thais

Francisco Junior, Wilmo

Galindo, Monica

Garcia, Lenise

Gatti, Sandra

Gauche, Ricardo

Giannella, Tais

Guimarães, Leandro B.

Hayashi, Maria

Igliori, Sonia

Justi, Rosária

Kondarzewski, Isabel

Labarce, Eliane

Laburú, Carlos

Lamas, Adriana

Lambach, Marcelo

Latini, Rose

Layrargues, Philippe

Leite, Bruno

Lima Junior, Paulo

Lima, Sorandra

Lima, Valderez M. R.

Longhini, lara

Lopes, Celi E.

Lorencini Júnior, Álvaro

Macedo, Elizabeth

Maia, Jorge S. S.

Maio, Eliane R.

Marcondes, Fabiane

Maricato, Fúlvia

Marques, Valéria

Martins, Lilian

Martins, Maria I.T. P.

Massi, Luciana

Mesquita, Nyuara
Universidade Federal da Bahia, Salvador, BA, Brasil

Universidade Estadual do Sudoeste da Bahia, Jequié, BA, Brasil

Universidade Federal do Rio Grande do Sul, Porto Alegre, RS, Brasil

Universidade Federal da Bahia, Salvador, BA, Brasil

Universidade Estadual de Campinas, Campinas, SP, Brasil

Universidade de São Paulo, Ribeirão Preto, SP, Brasil

Universidade Estadual de Feira de Santana, Feira de Santana, BA, Brasil

Universidade Federal de Ouro Preto, Ouro Preto, MG, Brasil

Universidade Federal do Rio Grande do Norte, Natal, RN, Brasil

Universidade Estadual de Campinas, Campinas, SP, Brasil

Universidade Federal de São Paulo, Diadema, SP, Brasil

Universidade Federal de Alagoas, Arapiraca, AL, Brasil

Universidade Estadual Paulista, São José do Rio Preto, SP, Brasil

Universidade de Brasília, Brasília, DF, Brasil

Universidade Estadual Paulista, Bauru, SP, Brasil

Universidade de Brasília, Brasília, DF, Brasil

Universidade Federal do Rio de Janeiro, Rio de Janeiro, RJ, Brasil

Universidade Federal de Santa Catarina, Florianópolis, SC, Brasil

Universidade Federal de São Carlos, São Carlos, SP, Brasil

Pontifícia Universidade Católica de São Paulo, São Paulo, SP, Brasil

Universidade Federal de Minas Gerais, Belo Horizonte, MG, Brasil

Universidade Estadual Paulista, Guaratinguetá, SP, Brasil

Universidade Estadual do Mato Grosso do Sul, Coxim, MS, Brasil

Universidade Estadual de Londrina, Londrina, PR, Brasil

Universidade Federal do $A B C$, Santo André, SP, Brasil

Universidade Tecnológica Federal do Paraná, Curitiba, PR, Brasil

Universidade Federal Fluminense, Niteró, Rj, Brasil

Universidade de Brasilia, Brasília, DF, Brasil

Universidade Federal Rural de Pernambuco, Recife, PE, Brasil

Universidade de Brasília, Brasília, DF, Brasil

Universidade Federal de Uberlândia, Uberlândia, MG, Brasil

Pontificia Universidade Catolica do Rio Grande do Sul, Porto Alegre, RS, Brasil

Universidade Federal de Uberlândia, Uberlândia, MG, Brasil

Universidade Cruzeiro do Sul, São Paulo, SP, Brasil

Universidade Estadual de Londrina, Londrina, PR, Brasil

Universidade do Estado do Rio de Janeiro, Rio de Janeiro, RJ, Brasil

Universidade Estadual do Norte do Paraná, Jacarezinho, PR, Brasil

Universidade Estadual de Maringá, Maringá, PR, Brasil

Instituto Federal de São Paulo, São José dos Campos, SP, Brasil

Universidade Estadual de Maringá, Maringá, PR, Brasil

Universidade Federal Rural do Rio de Janeiro, Seropédica, RJ, Brasil

Universidade de São Paulo, Ribeirão Preto, SP, Brasil

Universidade de Aveiro, Aveiro, Portugal

Universidade Estadual Paulista, Araraquara, SP, Brasil

Universidade Federal de Goiás, Goiânia, GO, Brasil 


\section{Janeiro a Setembro de 2020}

\section{Mohr, Adriana \\ Mól, Gerson}

Monteiro, Maria A.

Moraes, Andreia

Moreira, Plínio C.

Mozzer, Nilmara

Nascimento Júnior, Antônio

Nascimento, Silvania

Neiman, Zysman

Nicolli, Aline

Oliveira, Rosemary R.

Ornellas, Janaína

Orquiza-de-Carvalho, Lizete

Ovigli, Daniel

Passos, Marinez

Pedrini, Alexandre

Piassi, Luis

Pinhão, Francine

Pinheiro, Paulo C.

Pinto, Thiago Pedro

Pizarro, Mariana

Printes, Liane

Queiroz, Salete

Quintanilla-Gatica, Mario

Rabelo, Mauro

Razera, Júlio

Rezende Filho, Luiz

Ribeiro, Job

Rinaldi, Carlos

Ritter, Jaqueline

Rodrigues, Denise

Rosa, Katemari

Rosa, Maria I. F. P. S.

Salazar Lopez, Tatiana

Santana, Claudinei

Santos, André L. T. L.

Santos, Bruno F.

Santos, Flávia

Santos, João

Scheid, Neusa

Scucuglia, Ricardo

Silva, Antonio F. G.

Silva, Camila S.

Silva, Cibelle

Silva, Dirceu
Universidade Federal de Santa Catarina, Florianópolis, SC, Brasil

Universidade de Brasília, Brasília, DF, Brasil

Universidade Federal da Grande Dourados, Dourados, MS, Brasil

Centro Federal de Educação Tecnólogica Celso S. da Fonseca, Rio de Janeiro, RJ, Brasil

Universidade Federal de Ouro Preto, Ouro Preto, MG, Brasil

Universidade Federal de Ouro Preto, Ouro Preto, MG, Brasil

Universidade Federal de Lavras, Lavras, MG, Brasil

Universidade Federal de Minas Gerais, Belo Horizonte, MG, Brasil

Universidade Federal de São Paulo, São Paulo, SP, Brasil

Universidade Federal do Acre, Rio Branco, AC, Brasil

Universidade Estadual Paulista, Jaboticabal, SP, Brasil

Universidade Federal do Triângulo Mineiro, Uberaba, MG, Brasil

Universidade Estadual Paulista, Ilha Solteira, SP, Brasil

Universidade Federal do Triângulo Mineiro, Uberaba, MG, Brasil

Universidade Estadual de Londrina, Londrina, PR, Brasil

Universidade do Estado do Rio de Janeiro, Rio de Janeiro, RJ, Brasil

Universidade de São Paulo, São Paulo, SP, Brasil

Universidade do Estado do Rio de Janeiro, São Gonçalo, RJ, Brasil

Universidade Federal de São João del-Rei, São João del-Rei, MG, Brasil

Universidade Federal de Mato Grosso do Sul, Campo Grande, MS, Brasil

Instituto Federal do Paraná, Londrina, PE, Brasil

Universidade Federal de São Carlos, São Carlos, SP Brasil

Universidade de São Paulo, São Carlos, SP, Brasil

Pontificia Universidad Catolica de Chile, Santiago, Chile

Universidade de Brasilia, Brasília, DF, Brasil

Universidade Estadual do Sudoeste da Bahia, Jequié, BA, Brasil

Universidade Federal do Rio de Janeiro, Rio de Janeiro, RJ, Brasil

Universidade Federal do Triângulo Mineiro, Iturama, MG, Brasil

Universidade Federal de Mato Grosso, Cuiabá, MT, Brasil

Universidade Federal do Rio Grande, Rio Grande, RS, Brasil

Universidade do Estado do Rio de Janeiro, Rio de Janeiro, RJ, Brasil

Universidade Federal da Bahia, Salvador, BA, Brasil

Universidade Estadual de Campinas, Campinas, SP, Brasil

CINVESTAV Monterrey, Ciudad de Mexico, Mexico

Universidade Estadual do Sudoeste da Bahia, Vitória da Conquista, BA, Brasil

Colégio Pedro II, Rio de Janeiro, RJ, Brasil

Universidade Estadual do Sudoeste da Bahia, Jequié, BA, Brasil

Universidade Federal do Rio Grande do Sul, Porto Alegre, RS, Brasil

Universidade Federal de Mato Grosso do Sul, Campo Grande, MS, Brasil

Universidade Regional Integrada do Alto Uruguai e das Missões, Santo Ângelo, RS, Brasil

Universidade Estadual Paulista, São José do Rio Preto, SP, Brasil

Universidade Federal de São Carlos, São Carlos, SP, Brasil

Universidade Federal do Paraná, Curitiba, PR, Brasil

Universidade de São Paulo, São Carlos, SP, Brasil

Universidade Estadual de Campinas, Campinas, SP, Brasil 


\section{Janeiro a Setembro de 2020}

Silva, João A.

Silva, Josie A. P.

Silva, Leandro L.

Silva, Luciano

Silva, Messias

Silva, Silvia

Siqueira, Vera

Soares, Maria T. C.

Souza, Aguinaldo R.

Souza, Daniele C.

Souza, Luciana S.

Souza, Marcos L.

Souza, Thais S. N.

Strieder, Roseline

Struchiner, Miriam

Talamoni, Ana C.

Teixeira, Lucas A.

Teixeira, Odete P.

Teixeira, Paulo M. M.

Testoni, Leonardo

Valente, Jose Armando

Veraszto, Estéfano

Versuti, Fabiana

Vertuan, Rodolfo

Viana, Odaléa

Vianna, Carlos R.

Vianna, Deise M.

Zanata, Eliana

Zanon, Lenir

Zuffi, Edna
Universidade Federal do Rio Grande, Rio Grande, RS, Brasil Universidade Estadual de Ponta Grossa, Ponta Grossa, PR, Brasil Universidade Estadual Paulista, São José do Rio Preto, SP, Brasil Universidade Federal de Itajubá, Itajubá, MG, Brasil Universidade do Estado do Pará, Belém, PA, Brasil Universidade Estadual Paulista, Ilha Solteira, SP, Brasil Universidade Federal do Rio de Janeiro, Rio de Janeiro, RJ, Brasil Universidade Federal do Paraná, Curitiba, PR, Brasil Universidade Estadual Paulista, Bauru, SP, Brasil Universidade Federal do Triângulo Mineiro, Uberaba, MG, Brasil Universidade Estadual de Santa Cruz, Ilhéus, BA, Brasil Universidade Estadual do Sudoeste da Bahia, Jequié, BA, Brasil Universidade Federal do Estado do Rio de Janeiro, Rio de Janeiro, RJ, Brasil Universidade de Brasília, Brasília, DF, Brasil Universidade Federal do Rio de Janeiro, Rio de Janeiro, RJ, Brasil Universidade Estadual Paulista, São Vicente, SP, Brasil Universidade Estadual Paulista, Araraquara, SP, Brasil Universidade Estadual Paulista, Guaratinguetá, SP, Brasil Universidade Estadual do Sudoeste da Bahia, Jequi, BA, Brasil Universidade Federal de São Paulo, Diadema, SP, Brasil Universidade Estadual de Campinas, Campinas, SP, Brasil Universidade Federal de São Carlos, São Carlos, SP, Brasil Universidade de São Paulo, Ribeirão Preto, SP, Brasil Universidade Tecnologica Federal do Paraná, Toledo, PR, Brasil Universidade Federal de Uberlândia, Uberlândia, MG, Brasil Universidade Federal do Paraná, Curitiba, PR, Brasil Universidade Federal do Rio de Janeiro, Rio de Janeiro, RJ, Brasil Universidade Estadual Paulista, Bauru, SP, Brasil Universidade Regional do Noroeste do Estado do Rio Grande do Sul, ljui, RS, Brasil Universidade de São Paulo, São Carlos, SP, Brasil 